\title{
Anomalous refraction of self assembled gold nanowires studied by the generalized Snell's law
}

\author{
A. Belardini, ${ }^{* 1}$ G. Leahu, ${ }^{1}$ M. C. Larciprete, ${ }^{1}$ M. Centini, ${ }^{1}$ C. Sibilia, ${ }^{1}$ C. Martella, ${ }^{2}$ M. Giordano, ${ }^{2}$ D. Chiappe,${ }^{2}$ \\ F. Buatier de Mongeot ${ }^{2}$ \\ ${ }^{I}$ Dipartimento di Scienze di Base ed Applicate per l'Ingegneria. Sapienza Università di Roma, Via A. Scarpa 14, \\ 00161 Roma, Italy \\ ${ }^{2}$ Dipartimento di Fisica-Università di Genova and CNISM, Via Dodecaneso 33, 16146 Genova, Italy
}

Received May 12, 2013; accepted June 15, 2013; published June 30, 2013

\begin{abstract}
The generalized Snell's law of refraction was introduced by F. Capasso in 2011. One consequence of the law is that resonant metallic nanostructures at the interface between two dielectrics can bend the light in a controlled way. Alternatively, by measuring the bent of a beam of light induced by a nanopatterned metallic surface it is possible to retrieve information on the surface morphology. Here we show that deviation measurements of the light passing through curved metallic nanowires deposited on a glass slide gives information on the morphology only when resonance is excited.
\end{abstract}

By varying the relative phase shift of parts of the wave front of a beam of light it is possible to control the beam deviation. In common lenses it is possible to obtain this result by using different lengths of the optical path in different parts of the lens, thus the phase shift accumulates in thicknesses of the order of few millimeters. In 2011 F. Capasso and coworkers [1] showed that few nanometers of resonant metallic nanostructures at the interface between two dielectrics (labeled 1 and 2, respectively) can induce the phase discontinuity of a wavelength of light thus bending the beam that will refract by following the generalized Snell's law $[1,2,3]$ :

$$
\mathrm{n}_{2} \sin \phi_{2}-\mathrm{n}_{1} \sin \phi_{1}=\frac{\lambda_{0}}{2 \pi} \cdot \frac{d \Phi(x)}{d x},
$$

where $\mathrm{n}_{1}$ and $\mathrm{n}_{2}$ are the refractive indices of the dielectric 1 and 2 , respectively, $\lambda_{0}$ is the beam wavelength in vacuum and $\Phi(x)$ represents the phase discontinuity introduced by the nanostructure at the point $x$ of the interface. In this way it is possible to design very thin optical elements as aberration free lenses [4].

Here we use an alternative approach: it is possible to use the effect introduced by the generalized Snell's law as a tool in the characterization of nanopatterned plasmonic surfaces. Equation 1 states that, by measuring the refracted angle $\phi_{2}$ once it is known the incidence angle $\phi_{1}$, the gradient $d \Phi(x) / d x$ of the phase shift is also retrieved, important information on the morphology of the surface can be deducted.

\footnotetext{
*E-mail: alessandro.belardini@uniroma1.it
}

Here we report the measurements of the deviation of monochromatic light at $633 \mathrm{~nm}$ when it passes through a sample composed of self assembled bent gold nanowires (NWs) arrays with $125 \mathrm{~nm}$ of periodicity, deposited onto a nanopatterned glass slide.

The fabrication steps of the sample were fully described in [5-6] and in the following we summarize the procedure: a $0.14 \mathrm{~mm}$ thick soda-lime glass substrate was patterned by Ar+ ion beam sputtering as shown in Fig. 1a.

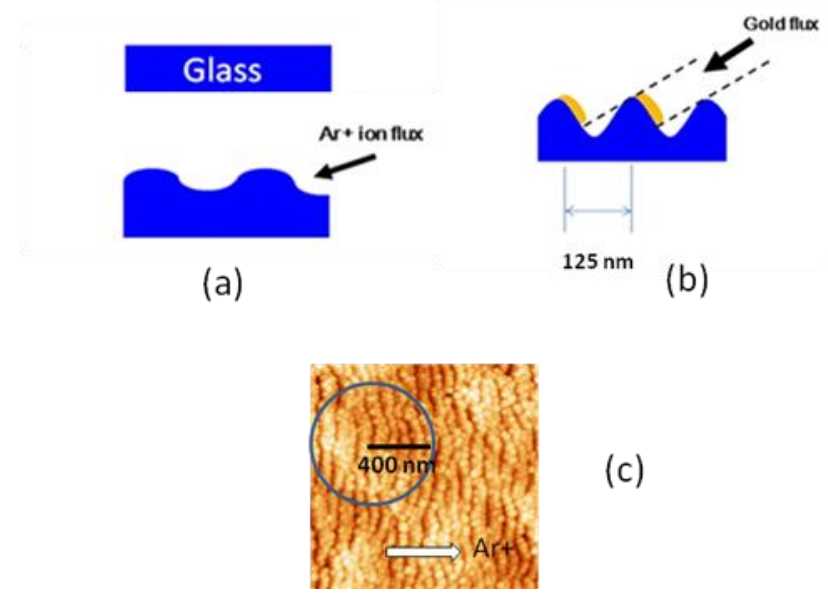

Fig. 1. The fabrication procedure: (a) first step, patterning of the glass with Ar+; (b) second step, gold evaporation; (c) AFM image of the final sample with nanowires.

The patterning forms periodic undulations (periodicity of $125 \mathrm{~nm}$ ) with the main wave-vector oriented parallel to the ion beam projection. The peak and the valleys of the undulations are curved with a radius of curvature $\mathrm{R}$ of around 400nm, which lies on the plan of the substrate and with a direction that is opposite to the Ar+ flux direction projection (see Fig.1c). The patterned glass was used as a substrate for gold evaporation. $40 \mathrm{~nm}$ of gold was thermally evaporated under grazing incidence condition $\left(80^{\circ}\right.$ respect to the normal) orthogonally to the ripple structures in order to localize metal agglomeration in correspondence to the convex side of the ridges (see fig.2c). The resulting sample is composed of connected 
polycrystalline gold curved wires of a diameter around $40-50 \mathrm{~nm}$ and $1-2 \mu \mathrm{m}$ long. In the transmission spectra reported in Ref.5, a broad absorption peak due to localised surface plasmon excitation is present around $600 \mathrm{~nm}$ only for the light that is polarised in the transverse direction with respect to the wires. The sample dimension is $1 \mathrm{~cm} \times 1 \mathrm{~cm}$ and it is fully patterned all over the surface and fully covered by gold nanowires except for a region of $5 \mathrm{~mm} \times 5 \mathrm{~mm}$ where the gold was removed in order to get a reference area.

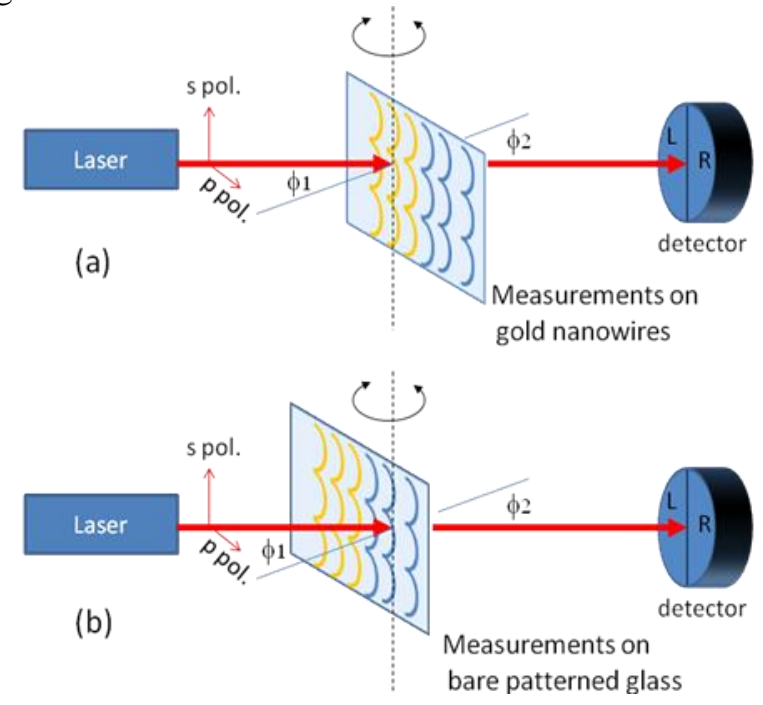

Fig. 2. Experimental setup: (a) measurements performed on the zone of the sample with gold nanowires; (b) reference measurements performed on the zone with bare nanopatterned glass (without gold nanowires). All the measurements are performed both in $\mathrm{s}$ and $\mathrm{p}$ polarization state.

The experimental setup is sketched in Fig. 2: in Fig. 2a the laser (that can be either in 's' or 'p' polarization state) impinges on the zone of the sample where the gold nanowires are present. The curvature of the wires lies mainly in the plane of incidence and when the polarization of the light is set in ' $p$ ' polarization state the plasmonic resonance is excited, on the contrary the plasmonic resonance was not excited when the polarization is set in ' $s$ '; in Fig. 2b the laser (that can be either in ' $s$ ' or ' $p$ ' polarization state) impinges on the zone of the sample where the gold nanowires were removed, thus it is present only in the bare patterned glass substrate. The last configuration is used as a reference for the deviation measurements. For both configurations the light source is a He-Ne laser emitting at $633 \mathrm{~nm}$, within the plasmonic absorption band of the sample. The light is then sent onto the sample at an incidence angle $\phi_{1}$ ranging in the $-40^{\circ} \div+40^{\circ}$ range. In order to retrieve the $\phi_{2}$ angle, the light at the output of the sample is detected by a photodiode divided in sectors (labeled $L$ for left side sector and $R$ for right side sector in Fig. 2) with a diameter of $8 \mathrm{~mm}$. The detector is posed at a distance $D=300 \mathrm{~mm}$ far away from the sample rotation axis. The output of the sensor was analysed by a position detector circuit giving the normalised difference between the power of the light in sector $L$ and the power of the light in sector $R$ :

$$
X=\frac{P_{L}-P_{R}}{P_{T}},
$$

where $P_{T}$ is the total power that impinges on the detector. The $X$ value is proportional to the displacement of the beam with respect to the centre of the detector and was supplied in volts at the output of the circuit. Measurements conversion from the circuit signal (in volts) to the real displacement (in $\mathrm{mm}$ ) is retrieved by a calibration measurement performed onto a glass slide of known thickness and results to be $0.05 \mathrm{~mm} / \mathrm{V}[5,7]$. Thus the refracted angle $\phi_{2}$ is :

$$
\phi_{2} \cong \frac{X}{D},
$$

that corresponds to a conversion factor between the $X$ value in volt and the angle in radiant of $1.6 \cdot 10^{-4} \mathrm{rad} / \mathrm{V}$.

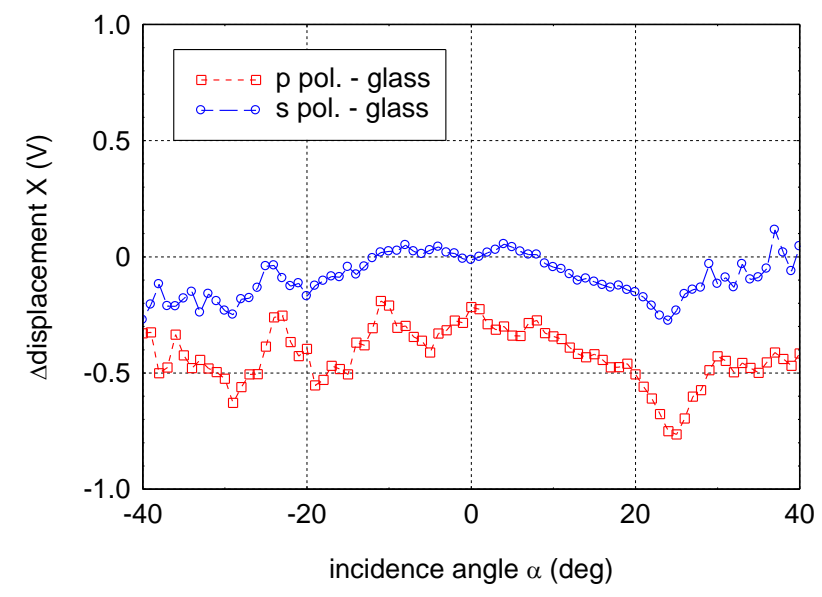

Fig. 3. Difference of the measured displacement $X$ on the zone of the sample with gold nanowires and the measured displacement measured on the bare patterned glass, for both 'p' polarized light (red squares) and 's 'polarized light (blue circles).

In Fig. 3 we show with blue circles, as a function of the incidence angle $\phi_{1}$, the difference between the displacement $X$ measured in the zone of the sample with nanowires and the displacement $X$ measured in the zone of the sample where the nanowires are removed (only the nanopatterned glass is present) with the laser set to ' $\mathrm{s}$ ' polarization state; meanwhile with the red squares we show the difference between the displacement $X$ measured in the zone of the sample with nanowires and the displacement $\mathrm{X}$ measured in the zone of the sample where the nanowires are removed (only the nanopatterned glass is present) with the laser set to 'p' polarization state. It is clear that when plasmonic resonance is not excited (' $s$ ' polarization) the deviation is similar to the one obtained by the bare patterned glass (the difference is close to zero for every incidence angle). Meanwhile with 
the polarization that excites the resonance (' $p$ ' polarization) the deviation is always different from zero and around the value of $-0.5 \mathrm{~V}$, which corresponds to an increment in the refracted angle of about $0.6 \cdot 10^{-4} \mathrm{rad}$.

It is the resonance condition that gives rise to a phase shift that produces a measurable deviation of the light. Here, instead of resonances obtained with features of the order of the wavelength (like, for example, in dielectric photonic band gap structures [8,9]), the metal itself gives rise to plasmonic resonance and few nanometers of thickness allows a good sensitivity in the visible wavelength scale $(633 \mathrm{~nm})$. In [5-6] we have shown the use of Eq. (1) can lead to the determination of the curvature direction of the nanowires. Previously, on similar samples of nanowires, we obtained similar results on curvature and sub-wavelength features detection by using more complex second harmonic generation (SHG) set-ups $[10,11]$.

In conclusion, we investigated curved gold nanowires deposited onto a glass slide by means of a deviation measurements setup. Despite the fact that the section of the wires (around 50nm) and their periodicity $(125 \mathrm{~nm})$ are comparably smaller than the wavelength used in the measurement $(633 \mathrm{~nm})$, the deviation is appreciable in resonance condition because of the generalised Snell's law, which allows a different refracted angle with respect to the classical Snell's law when the resonance produces a macroscopic phase shift.

This work was partially financed by PRIN-MIUR 2008J858Y7, by MARINE project granted by Italian Ministry of Defense, by Fondazione Carige and by ENEA under project MSE.

\section{References}

[1] N. Yu, P. Genevet, M.A. Kats, F. Aieta, J.-P. Tetienne, F. Capasso, Z. Gaburro, Science 334, 333 (2011).

[2] X. Ni, N.K. Emani, A.V. Kildishev, A. Boltasseva, V.M. Shalaev, Science 335, 427 (2012).

[3] N. Engheta, Science 334, 317 (2011).

[4] F. Aieta, P. Genevet, M.A. Kats, N. Yu, R. Blanchard, Z. Gaburro, F. Capasso, Nano Lett. 12, 4932 (2012).

[5] A. Belardini, F. Pannone, G. Leahu, M.C. Larciprete, M. Centini, C. Sibilia, C. Martella, M. Giordano, D. Chiappe, F. Buatier de Mongeot, Appl. Phys. Lett. 100, 251109 (2012).

[6] A. Belardini, F. Pannone, G. Leahu, M.C. Larciprete, M. Centini, C. Sibilia, C. Martella, M. Giordano, D. Chiappe, F. Buatier de Mongeot, J. Europ. Opt. Soc. Rap. Public. 7, 12051 (2012).

[7] A. Belardini, C. Sibilia, Optoelectron. Adv. Mater. - Rapid Commun. 7, 184 (2013).

[8] A. Sinibaldi, N. Danz, E. Descrovi, P. Munzert, U. Schulz, F. Sonntagd, L. Dominici, F. Michelotti, Sensors and Actuators B 174, 292-298 (2012).

[9] A. Belardini, A. Bosco, G. Leahu, M. Centini, E. Fazio, C. Sibilia, M. Bertolotti, S. Zhukovsky, S.V. Gaponenko, Appl. Phys. Lett. 89, 031111 (2006).
[10] A. Belardini, M.C. Larciprete, M. Centini, E. Fazio, C. Sibilia, D. Chiappe, C. Martella, A. Toma, M. Giordano, F. Buatier de Mongeot, Phys. Rev. Lett. 107, 257401 (2011).

[11] A. Belardini, M.C. Larciprete, M. Centini, E. Fazio, C. Sibilia, M. Bertolotti, A. Toma, D. Chiappe, F. Buatier de Mongeot, Opt. Expr. 17, 3603 (2009). 